\title{
Intersection Reverse Sequences and Geometric Applications
}

\author{
Adam Marcus ${ }^{1, \star}$ and Gábor Tardos ${ }^{2, \star \star}$ \\ 1 Department of Mathematics, Georgia Institute of Technology, \\ Atlanta, GA 30332-0160 \\ adam@math.gatech.edu \\ 2 Alfréd Rényi Institute of Mathematics, \\ Pf.127, H-1364 Budapest, Hungary \\ tardos@renyi.hu
}

\begin{abstract}
Pinchasi and Radoičić [1] used the following observation to bound the number of edges in a topological graph with no self-intersecting cycles of length 4 : if we make a list of the neighbors for every vertex in such a graph and order these lists cyclicly according to the connecting edge, then the common elements in any two lists have reversed cyclic order. Building on their work we give an estimate on the size of the lists having this property. As a consequence we get that a topological graph on $n$ vertices not containing a self-intersecting $C_{4}$ has $O\left(n^{3 / 2} \log n\right)$ edges. Our result also implies that $n$ pseudo-circles in the plane can be cut into $O\left(n^{3 / 2} \log n\right)$ pseudo-segments, which in turn implies bounds on pointcurve incidences and on the complexity of a level of an arrangement of curves.
\end{abstract}

\section{Introduction}

In this paper we consider cyclicly ordered sequences of distinct symbols from a finite alphabet. We say that two such sequences are intersection reverse if the common elements appear in reversed cyclic order in the two sequences.

A topological graph is a graph without loops or multiple edges drawn in the plane (vertices correspond to distinct points, edges correspond to Jordan curves connecting the corresponding vertices). We assume no edge passes through a vertex other than its endpoints and every two edges have a finite number of common interior points and they properly cross at each of these points. For a vertex $v$ of a topological graph $G$ let $L_{G}(v)$ be the list of its neighbors ordered cyclicly counterclockwise according to the initial segment of the connecting edge.

Pinchasi and Radoičić [1] noticed the following simple fact:

Fact 1. If the lists $L_{G}(u)$ and $L_{G}(v)$ are not intersection reverse for all distinct vertices $u$ and $v$ of the topological graph $G$, then $G$ contains a self-crossing cycle

\footnotetext{
* Research was done while a Visiting Researcher at The Alfréd Rényi Institute, Budapest and was supported by the Fulbright Program in Hungary.

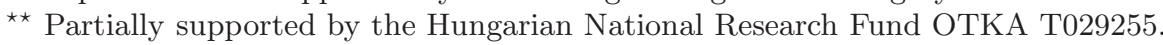


of length 4. Moreover, $u$ and $v$ are opposite vertices of a cycle of length 4 in $G$ that has two edges that cross an odd number of times.

For the proof one only has to consider drawings of the complete bipartite graph $K_{2,3}$ (see details in [1]). Pinchasi and Radoičić used Fact 1 to bound the number of edges of a topological graph not containing a self-crossing $C_{4}$. They showed that such a graph on $n$ vertices has $O\left(n^{8 / 5}\right)$ edges. Following in their footsteps, we use the same property to improve their bound to $O\left(n^{3 / 2} \log n\right)$. This bound is tight apart from the logarithmic factor as one has (abstract) simple graphs on $n$ vertices with $\Omega\left(n^{3 / 2}\right)$ edges containing no $C_{4}$-subgraph. Our main technical result is the following:

Theorem 1. Let us be given $m$ cyclicly ordered lists of $d$ element subsets of a set of $n$ symbols. If the lists are pairwise intersection reverse, then

$$
d=O\left(\sqrt{n} \log n+\frac{n}{\sqrt{m}}\right) .
$$

We give the proof of this theorem in Section 2. In Section 3 we prove several consequences, among them the following bound:

Theorem 2. If an n-vertex topological graph does not contain a self-crossing $C_{4}$ it has $O\left(n^{3 / 2} \log n\right)$ edges. The same holds if every pair of edges in every $C_{4}$ subgraph crosses an even number of times.

The most important consequence of Theorem 1 deals with collections of pseudo-circles: simple closed Jordan curves, any two of which intersect at most twice, with proper crossings at each intersection. The result readily generalizes to unbounded open curves such as pseudo-parabolas, the graphs of continuous real functions defined on the entire real line such that any two intersect at most twice and they properly cross at these intersections.

Tamaki and Tokuyama [2] were the first to consider the problem of cutting pseudo-parabolas into pseudo-segments, i.e., subdividing the original curves into segments such that any two segments intersect at most once. Such a separation turns out to be quite useful since pseudo-segments are much easier to work with than pseudo-parabolas and pseudo-circles, as will be seen in Section 3.

Tamaki and Tokuyama [2] proved that $n$ pseudo-parabolas can be cut into $O\left(n^{5 / 3}\right)$ pseudo-segments. This was extended to $x$-monotone pseudo-circles by Aronov and Sharir [3] and by Agarwal, et al. [4]. It was also improved for certain collections of curves that admit a three parameter algebraic parameterization to $n^{3 / 2} \log ^{\alpha^{O(1)}(n)}(n)$, where $\alpha$ is the inverse Ackermann function.

Previously, the best bound for arbitrary pseudo-parabolas and $x$-monotone pseudo-circles was $O\left(n^{8 / 5}\right)$ [4], which uses the result of Pinchasi and Radoičić on topological graphs without self-crossing $C_{4}$. With our improvement of the latter result, we can prove that $n$ pseudo-parabolas can be cut into $O\left(n^{3 / 2} \log n\right)$ pseudo-segments. This substantially improves the previous bounds for arbitrary collections and is still slightly better than results on families with algebraic parameterization; we replace a little-over-polylog factor with a single log factor. In 
doing so, we are able to simplify the results in $[4,1,2]$, as well as generalize them to the cases when the pseudo-parabolas and pseudo-circles are not necessarily $x$-monotone.

In Section 3 we show the above result, as well as its applications to pointcurve incidences and the level complexities of curve arrangements. See $[4,3,5,6$, 2] for more details and applications.

Finally in Section 4 we discuss a few related problems that are still open. All logarithms in this paper are binary.

\section{Intersection Reverse Sequences}

In this section we prove our main technical result, Theorem 1. Much of the proof follows the argument of Pinchasi and Radoičić [1]. We start with an overview of their techniques.

Pinchasi and Radoičić break the cyclicly ordered lists into linearly ordered blocks. They consider pairs of blocks from separate lists and pairs of symbols contained in both blocks. They distinguish between same pairs and different pairs according to whether the two symbols appear in the same or in different order. They observe that any pair of symbols that appears in many blocks must produce almost as many same pairs as different pairs. On the other hand the intersection reverse property forces two cyclicly ordered lists - unless most of their intersection is concentrated into a single pair of blocks - to contribute many more different than same pairs. Exceptional pairs of cyclicly ordered lists are treated separately with techniques from extremal graph theory. They optimize in their choice for the length of the blocks.

We follow almost the same path, but instead of optimizing for block length we consider many block lengths (an exponential sequence) simultaneously. For two intersection reverse lists no block length yields significantly more same pairs than different pairs, but we will show that some block length actually gives many more different pairs than same pairs. As a consequence we do not have to bound "exceptional pairs" of lists separately.

Definition. We will use the term sequence to denote a linearly ordered list of distinct symbols and the term cyclic sequence to denote a cyclicly ordered list of distinct symbols. Clearly, if one breaks up a cyclic sequence into blocks, then the blocks are (linearly ordered) sequences. For a sequence or cyclic sequence $A$ we write $\bar{A}$ for the set of symbols in $A$. We define intersection reverse for sequences just as for cyclic sequences: we say that the sequences $A$ and $B$ are intersection reverse if they induce inverse linear orders on $\bar{A} \cap \bar{B}$. If two sequences are not intersection reverse, we call them singular. Note that if two sequences $A$ and $B$ have $|\bar{A} \cap \bar{B}| \leq 1$, then the sequences are trivially intersection reverse. The same holds for cyclic sequences $A$ and $B$ if $|\bar{A} \cap \bar{B}| \leq 2$.

For a sequence $B$ and symbols $a \neq b$ we define

$$
f(B, a, b)=\left\{\begin{aligned}
0 & \text { if } a \notin \bar{B} \text { or } b \notin \bar{B}, \\
1 & \text { if } a \text { precedes } b \text { in } B, \\
-1 & \text { if } b \text { precedes } a \text { in } B .
\end{aligned}\right.
$$


For two sequences $B$ and $B^{\prime}$ we let $f\left(B, B^{\prime}, a, b\right)=f(B, a, b) f\left(B^{\prime}, a, b\right)$. Notice that $f\left(B, B^{\prime}, a, b\right)=1$ for same pairs and $f\left(B, B^{\prime}, a, b\right)=-1$ for different pairs, and that $\sum f\left(B, B^{\prime}, a, b\right)$ corresponds to the difference between the number of same pairs and different pairs.

The next lemma is taken from [1]. The summation $\sum_{a \neq b}$ here and later in this section is taken for all ordered pair of distinct symbols $a$ and $b$.

Lemma 3. Let the cyclic sequences $A$ and $A^{\prime}$ consist of the (linearly ordered) blocks $B_{1}, \ldots, B_{k}$ and $B_{1}^{\prime}, \ldots, B_{k^{\prime}}^{\prime}$, respectively. If $A$ and $A^{\prime}$ are intersection reverse, then at most one of the pairs $B_{i}, B_{j}^{\prime}$ is singular. For this singular pair we have

$$
\sum_{a \neq b} f\left(B_{i}, B_{j}^{\prime}, a, b\right) \leq\left|\overline{B_{i}} \cap \overline{B_{j}^{\prime}}\right| .
$$

For the rest of this section, let $A^{1}, \ldots, A^{m}$ be the $m$ cyclic sequences in the theorem, $p^{i j}=\left|\overline{A^{i}} \cap \overline{A^{j}}\right|$, and $p=\sum_{i \neq j} p^{i j}$. The following bounds follow directly from the inequality between the arithmetic and quadratic means assuming $d m>2 n$ (otherwise the statement of Theorem 1 is immediate).

Lemma 4. We have $p \geq \frac{d^{2} m^{2}}{2 n}$ and $\sum_{i \neq j}\left(p^{i j}\right)^{2} \geq \frac{p^{2}}{m^{2}}$.

We split each $A^{i}$ into two almost equal size consecutive blocks $A_{0}^{i}$ and $A_{1}^{i}$. In general, for a $0-1$ sequence $s$ we split the block $A_{s}^{i}$ of $A^{i}$ into two almost equal halves (differing in size by at most 1): $A_{s 0}^{i}$ and $A_{s 1}^{i}$. The cyclic order of $A^{i}$ linearly orders all of these blocks. Let $k=\lceil\log d\rceil<\log n+1$. Clearly, any $0-1$ sequence $s$ of length $k$ satisfies $\left|\overline{A_{s}^{i}}\right| \leq 1$.

For $1 \leq i \leq m$ and $1 \leq j \leq m$ we let

$$
S^{i j}=\sum_{a \neq b} \sum_{l=1}^{k} \sum_{|s|=|t|=l} w_{l} f\left(A_{s}^{i}, A_{t}^{j}, a, b\right),
$$

where the summation is taken over all pairs of symbols $a \neq b$, and $0-1$ sequences $s$ and $t$ of size $l$. The weights $w_{l}$ in the formula are positive and we set them later. Our goal is to contrast a lower bound on $\sum_{i \neq j} S^{i j}$ (or rather on the partial sum for fixed symbols $a \neq b$ ) with upper bounds on the individual $S^{i j}$. The pair $(i, j)$ (just as the pair $(a, b)$ above) is considered ordered resulting in double (quadruple, really) counting.

The lower bound is straightforward:

Lemma 5. $\sum_{i \neq j} S^{i j} \geq-m d^{2} \sum_{l=1}^{k} \frac{w_{l}}{2^{l}}$

Proof. Notice that for fixed $a, b$, and length $|s|=|t|$ we get a perfect square when summing over all $i$ and $j$. In particular,

$$
\sum_{i=1}^{m} \sum_{j=1}^{m} S^{i j}=\sum_{l=1}^{k} w_{l} \sum_{a \neq b}\left(\sum_{i=1}^{m} \sum_{|s|=l} f\left(A_{s}^{i}, a, b\right)\right)^{2} \geq 0 .
$$


We can bound the $S^{i i}$ terms separately as they are merely the weighted sum of the number of pairs in the blocks:

$$
\sum_{i \neq j} S^{i j}=\sum_{i=1}^{m} \sum_{j=1}^{m} S^{i j}-\sum_{i=1}^{m} S^{i i} \geq 0-m d^{2} \sum_{l=1}^{k} \frac{w_{l}}{2^{l}}
$$

The upper bound, however, requires more effort.

Lemma 6. For $i \neq j$ we have

$$
S^{i j} \leq p^{i j} \sum_{l=1}^{k} w_{l}-\frac{\left(p^{i j}\right)^{2}}{4 \sum_{l=1}^{k} \frac{1}{w_{l}}} .
$$

Proof. We fix the indices $i \neq j$ and consider the following quantities:

$$
\begin{aligned}
r_{s t} & =\left|\overline{A_{s}^{i}} \cap \overline{A_{t}^{j}}\right| \text { and } \\
Q_{s t} & =\sum_{a \neq b} f\left(A_{s}^{i}, A_{t}^{j}, a, b\right)
\end{aligned}
$$

where $s$ and $t$ are $0-1$ sequences of equal length.

For a fixed $1 \leq l \leq k$ the blocks $A_{s}^{i}$ with $|s|=l$ form a subdivision of $A^{i}$, while the blocks $A_{t}^{j}$ with $|t|=l$ form a subdivision of $A^{j}$. We use Lemma 3: there is at most one singular pair $\left(A_{s}^{i}, A_{t}^{j}\right)$ for every length $|s|=|t|=l$. For these singular pairs we have $Q_{s t} \leq r_{s t}$, while for the intersection reverse ones straightforward calculation gives $Q_{s t}=r_{s t}-r_{s t}^{2}$. Note that for $|s|=|t|=k$ the corresponding pairs are trivially intersection reverse.

For a $0-1$ sequence $s$ of length $|s|>1$ let $s^{\prime}$ denote the sequence obtained from $s$ by deleting its last digit; hence $A_{s^{\prime}}^{i}$ contains $A_{s}^{i}$. We call a pair $(s, t)$ of equal length $0-1$ sequences a leader if $\left(A_{s}^{i}, A_{t}^{j}\right)$ is intersection reverse and either $|s|=|t|=1$ or the pair $\left(A_{s^{\prime}}^{i}, A_{t^{\prime}}^{j}\right)$ is singular. Clearly, there are at most 4 leaders in any given length. Furthermore, any symbol $a \in \overline{A^{i}} \cap \overline{A^{j}}$ is contained in $\overline{A_{s}^{i}} \cap \overline{A_{t}^{j}}$ for exactly one leader pair $(s, t)$ : the longest intersection reverse pair of blocks containing them (recall that we only consider pairs of blocks with equal length subscripts). Thus we have $\sum_{(s, t) \in L} r_{s t}=p^{i j}$ for the set $L$ of leader pairs.

We use $Q_{s t}=r_{s t}-r_{s t}^{2}$ for leader pairs $(s, t)$ only. For all other pairs, intersection reverse or singular, we use $Q_{s t} \leq r_{s t}$ :

$$
S^{i j} \leq \sum_{l=1}^{k} w_{l} \sum_{|s|=|t|=l} r_{s t}-\sum_{(s, t) \in L} w_{|s|} r_{s t}^{2}=p^{i j} \sum_{l=1}^{k} w_{l}-\sum_{(s, t) \in L} w_{|s|} r_{s t}^{2}
$$

since $\sum_{|s|=|t|=l} r_{s t}=p^{i j}$ for any fixed $l$. The Cauchy-Schwarz inequality gives

$$
\left(\sum_{(s, t) \in L} w_{|s|} r_{s t}^{2}\right)\left(\sum_{(s, t) \in L} \frac{1}{w_{|s|}}\right) \geq\left(\sum_{(s, t) \in L} r_{s t}\right)^{2}=\left(p^{i j}\right)^{2} .
$$


Here $\sum_{(s, t) \in L}\left(1 / w_{|s|}\right) \leq 4 \sum_{l=1}^{k}\left(1 / w_{l}\right)$, so we conclude that

$$
S^{i j} \leq p^{i j} \sum_{l=1}^{k} w_{l}-\frac{\left(p^{i j}\right)^{2}}{4 \sum_{l=1}^{k} \frac{1}{w_{l}}}
$$

as claimed.

Comparing the two estimates in the previous lemmas gives the theorem.

Proof (of Theorem 1). Using Lemmas 5, 6, and 4 (respectively) we obtain

$$
\begin{aligned}
-m d^{2} \sum_{l=1}^{k} \frac{w_{l}}{2^{l}} & \leq \sum_{i \neq j} S^{i j} \leq \sum_{i \neq j} p^{i j} \sum_{l=1}^{k} w_{l}-\frac{\sum_{i \neq j}\left(p^{i j}\right)^{2}}{4 \sum_{l=1}^{k} \frac{1}{w_{l}}} \\
& \leq p \sum_{l=1}^{k} w_{l}-\frac{p^{2}}{4 m^{2} \sum_{l=1}^{k} \frac{1}{w_{l}}}
\end{aligned}
$$

Using the fact that $p \geq d^{2} m^{2} /(2 n)$ from Lemma 4 we get

$$
d \leq 4 \sqrt{n} \sqrt{\sum_{l=1}^{k} w_{l} \sqrt{\sum_{l=1}^{k} \frac{1}{w_{l}}}}
$$

or

$$
d \leq \frac{6 n}{\sqrt{m}} \sqrt{\sum_{l=1}^{k} \frac{w_{l}}{2^{l}}} \sqrt{\sum_{l=1}^{k} \frac{1}{w_{l}}} .
$$

We choose the weights $w_{l}$ now. Equal weights $\left(w_{l}=1\right)$ yield $d=O(\sqrt{n} \log n+$ $n \sqrt{\log n} / \sqrt{m})$, but we can improve on this bound by choosing

$$
w_{l}=\frac{1}{1+\frac{k}{2^{l / 2}}} .
$$

In this case $\sum_{l=1}^{k} w_{l} \leq k, \sum_{l=1}^{k}\left(1 / w_{l}\right) \leq 4 k$, and $\sum_{l=1}^{k}\left(w_{l} / 2^{l}\right) \leq 3 / k$. Thus we either have $d \leq 8 k \sqrt{n}$ or $d \leq 21 n / \sqrt{m}$ and the theorem follows.

\section{Consequences}

In this section we present several geometric applications of Theorem 1.

\subsection{Self-intersecting Cycles of Length 4}

Any bound for the $n=m$ case of Theorem 1 carries over to the number of edges of a topological graph not containing self-intersecting $C_{4}$ by [1]. Using the following corollary, however, the proof is even simpler: 
Corollary 7. Let us be given $m$ cyclic sequences over an n-element set of symbols. If the cyclic sequences are pairwise intersection reverse, then the sum of their sizes is $O(m \sqrt{n} \log n+n \sqrt{m})$.

The simple deduction from Theorem 1 is omitted.

The proof of Theorem 2 is now straightforward. The statements are direct consequences of Corollary 7 using Fact 1. Notice that the sum of the sizes of the lists of neighbors (the sum of the degrees) is twice the number of edges.

\subsection{Cutting Number}

Tamaki and Tokuyama [2] considered the cutting number of a collection of curves. This is the number of cuts needed to obtain a collection of shorter curves, each pair of which intersects at most once.

The restriction of the next corollary to so called $x$-monotone pseudo-circles can be derived from Theorem 2 using a combination of techniques in the papers $[4,2]$. A simple and direct deduction from Corollary 7 that does not require any additional assumption on the pseudo-circles will appear in the final version of this paper. Recall that this result slightly improves the best previous bound for ( $x$-monotone) pseudo-circles with a three parameter algebraic representation as defined in [4] (such as circles or axis-aligned parabolas) and substantially improves the previous bounds for pseudo-circles lacking such representation. For the definition of pseudo-circles see Section 1.

Corollary 8. An arrangement of $n$ pseudo-circles can be cut at $O\left(n^{3 / 2} \log n\right)$ points such that the resulting curves form a system of pseudo-segments.

Corollary 8 naturally generalizes to collections of open Jordan curves including, for example, pseudo-parabolas. We omit the straightforward deduction. We call a collection of simple closed and open Jordan curves a generalized pseudocircle collection if both ends of every open curve are at infinity, any two curves have at most two points of intersection, and the curves cross properly at each intersection.

Corollary 9. A generalized pseudo-circle collection $\mathcal{C}$ of $n$ curves can be cut at $O\left(n^{3 / 2} \log n\right)$ points such that the resulting curve segments form a system of pseudo-segments.

\subsection{Levels}

Corollary 9 also has many consequences in the study of levels in arrangements of curves. Tamaki and Tokuyama [2] first showed the usefulness of cutting numbers in this area, and progress has been made by Chan $[5,6]$.

Definition. Let $\mathcal{C}$ be the set of the graphs of the real functions $f_{1}, f_{2}, \ldots, f_{n}$. We assume that each $f_{i}$ is continuous and defined everywhere on the real line, and that any pair of curves in $\mathcal{C}$ intersects a finite number of times. We define the $k^{\text {th }}$ level of $\mathcal{C}$ to be the closure of the locus of points $(x, y)$ on the curves in $\mathcal{C}$ with $\left|\left\{i: f_{i}(x) \leq y\right\}\right|=k$. The $k^{t h}$ level consists of portions of the curves in $\mathcal{C}$, 
delimited by intersections between these curves. We will call the total number of curve segments in a level its complexity.

Chan [6] derives an upper bound on the complexity of a given level using the number of cuts needed to turn a collection of pseudo-parabolas into pseudosegments. Our Corollary 9 improves his analysis. We omit the details.

Corollary 10. Let $\mathcal{C}$ be a collection of $n$ pseudo-parabolas. The maximum complexity of any level of $\mathcal{C}$ is $O\left(n^{3 / 2} \log ^{2} n\right)$.

The above corollary represents a substantial improvement over the previous bound in [6] for an arbitrary collection of pseudo-parabolas. For a collection possessing a three parameter algebraic representation (as defined in [4]) the improvement is marginal, replacing a little-over-polylog term by $\log ^{2} n$. These improvements carry over to levels of arrangements of algebraic curves of degree higher than two by the technique of bootstrapping, as developed in [6]. We do not state the slightly improved bounds here.

\subsection{Incidences and Faces}

Aronov and Sharir [3] also used cutting numbers in their analysis of incidences between curves and points in the plane.

Definition. Let $\mathcal{C}$ be a set of curves and $P$ a set of points in the plane. We define $I(\mathcal{C}, \mathcal{P})$ to be the number of incidences between $\mathcal{C}$ and $\mathcal{P}$, that is the number or pairs $(c, p) \in \mathcal{C} \times \mathcal{P}$ such that curve $c$ contains point $p$. We also define $K(\mathcal{C}, \mathcal{P})$ to be the sum of the complexities of the faces of the arrangement $\mathcal{C}$ containing points in $P$ (assuming now that they are not on the curves). The complexity of a face is defined to be the number of curve segments that comprise the face.

The results in [3] relate the values of $K(\mathcal{C}, \mathcal{P})$ and $I(\mathcal{C}, \mathcal{P})$ to the cutting numbers discussed above. Applying their results and Corollary 9 , we get

Corollary 11. If $\mathcal{C}$ is a collection of $n$ generalized pseudo-circles and $P$ a set of $m$ points, then

1. $I(\mathcal{C}, \mathcal{P})=O\left(m^{2 / 3} n^{2 / 3}+m+n^{3 / 2} \log n\right)$

2. $K(\mathcal{C}, \mathcal{P})=O\left(m^{2 / 3} n^{2 / 3}+m+n^{3 / 2} \log ^{3} n\right)$

\section{Open Problems}

The results in this paper raise a number of interesting questions. Theorem 2 is tight except possibly for the logarithmic factor as graphs with $n$ vertices and $\Omega\left(n^{3 / 2}\right)$ edges are known which do not contain any $C_{4}$. This also implies that the $n=m$ special case of Theorem 1 and Corollary 7 are almost tight. Nevertheless, it would be interesting to know if the logarithmic factor is needed.

Problem 1. Is the logarithmic factor needed in Theorem 2?

The geometric consequences use Theorem 1 in the $n=m$ special case, but it is interesting to give bounds in the asymmetric cases as well. We define $R(n, m)$ to be the maximum total length of $m$ pairwise intersection reverse cyclic sequences 
over an alphabet of size $n$. With this notation Corollary 7 gives $R(n, m)=$ $O(m \sqrt{n} \log n+n \sqrt{m})$. We collect here a few simple lower and upper bounds for $R(n, m)$.

A trivial consequence of the property that a collection of cyclic sequences are pairwise intersection reverse is that no three symbols appear together in three cyclic sequences. By the Kővári-T. Sós-Turán Theorem [7], we have that $R(n, m)=O\left(n m^{2 / 3}+m\right)$ and $R(n, m)=O\left(n^{2 / 3} m+n\right)$. The first bound supersedes the bound in Corollary 7 if $m \geq n^{3 / 2}$. The second bound supersedes the bound in Corollary 7 if $m<n^{2 / 3}$. So for these extremely large or small values of $m$ Corollary 7 is not tight.

The simplest constructions of intersection reverse cyclic sequences are constructions for collections of subsets intersecting each other in at most two elements. No matter how we order these subsets the resulting collection of cyclic sequences is pairwise intersection reverse. A simple construction for such subsets is any collection of circles in the plane over a finite field where -1 is not a square. Taking all points of the plane and a subset of the circles gives $R(n, m)=\Omega(m \sqrt{n})$ for $m \leq n^{3 / 2}$. Taking all circles and a subset of the points gives $R(n, m)=\Omega\left(n m^{2 / 3}\right)$ for $m \geq n^{3 / 2}$. A collection of singleton sets gives the trivial bound $R(n, m) \geq m$, which is better than the previous bounds for $m>n^{3}$. Pairwise disjoint sets provide the other trivial $R(n, m) \geq n$ bound, which is better than the other bounds for $m \leq \sqrt{n}$.

The solid lines in the logarithmic scale diagram in Figure 1 shows the lower and upper bounds mentioned above. These bounds determine $R(n, m)$ up to a constant factor for $m \geq n^{3 / 2}$ and $m \leq n^{1 / 3}$ and up to a logarithmic factor for $n \leq m \leq n^{3 / 2}$. In any construction proving better lower bounds then the ones above, a typical pair of cyclic sequences will need to intersect in many elements, so the cyclic order is essential in those constructions. We present such a construction below proving $R(n, m)=\Omega\left(n^{5 / 6} m^{1 / 2}\right)$ for $n^{1 / 3}<m<n^{2 / 3}$. This bound is represented in Figure 1 by the dashed line. The area of "uncertainty" is shaded. Even with this construction, the upper and lower bounds for $R(n, m)$ are far apart for $n^{1 / 3}<m<n$.

Construction. This construction is based on the construction of Gy. Elekes [8] of a set of axis-aligned parabolas and a set of points with a large number of incidences. Observe that axis-aligned parabolas form a collection of pseudo-parabolas: any pair intersects at most twice. For integers $b \geq a \geq 1$ consider the subset $P=\left\{(i, j):|i| \leq a,|j| \leq 3 a^{2} b\right\}$ of the integer grid and consider the collection $\mathcal{C}$ of parabolas (and lines) given by $y=u x^{2}+v x+w$ with integers $u, v$, and $w$ satisfying $|u| \leq b,|v| \leq a b$ and $|w| \leq a^{2} b$. We have $m=|P|=$ $(2 a+1)\left(6 a^{2} b+1\right)=\Theta\left(a^{3} b\right)$ and $n=|\mathcal{C}|=(2 b+1)(2 a b+1)\left(2 a^{2} b+1\right)=\Theta\left(a^{3} b^{3}\right)$. Clearly, each curve in $\mathcal{C}$ contains a point in $P$ for each possible $x$ coordinate, a total of $2 a+1$ points. For each $p \in P$ we define the linearly ordered list $B_{p}$ of all the curves in $\mathcal{C}$ passing through $p$. We order the list $B_{p}$ according to the slopes of the curves at $p$ (breaking ties arbitrarily). As a result we get $m$ linearly ordered lists of subsets of the set of $n$ symbols. It is easy to verify that these lists are intersection reverse. Their total length is the number of incidences between $P$ and $\mathcal{C}$, which is $\Theta\left(a^{4} b^{3}\right)=\Theta\left(n^{5 / 6} m^{1 / 2}\right)$. 


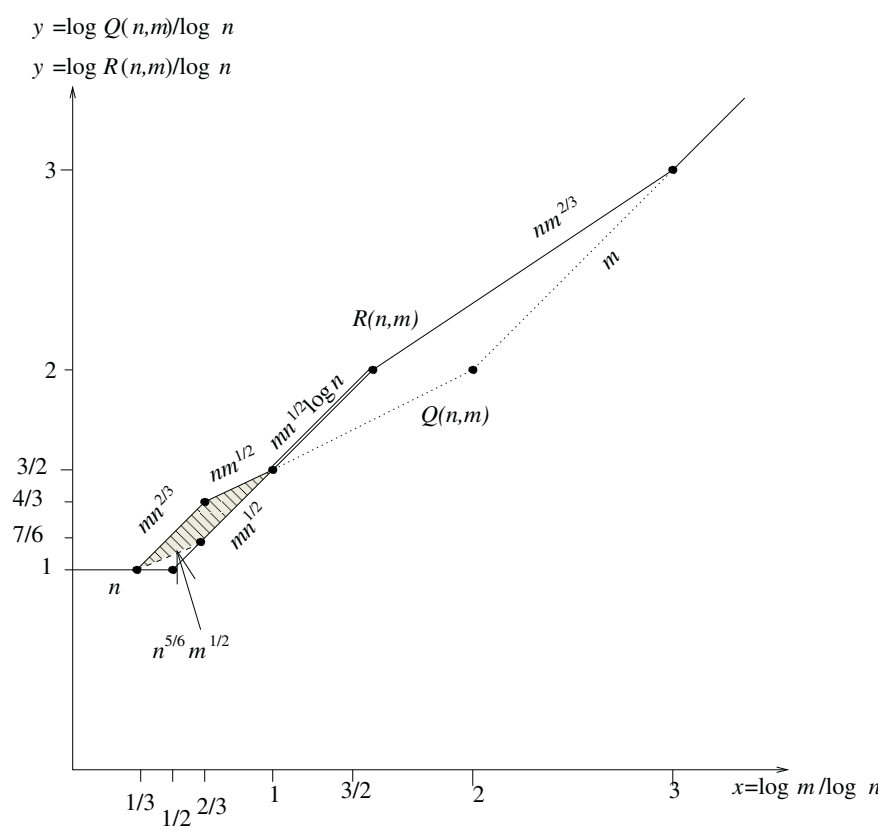

Fig. 1. Bounds and area of uncertainty for $R(n, m)$ and $Q(n, m)$.

Problem 2. Is it possible to find $n^{2 / 3}$ pairwise intersection reverse cyclic sequences over an alphabet of size $n$ such that their total lengths sum to significantly more than $n^{7 / 6}$ ?

Note that for $m=n^{2 / 3}$ both constructions gives cyclic sequences with total size $\Theta\left(n^{7 / 6}\right)$. One of the constructions is based on finite geometry, the other on Euclidean geometry. It seems to be hard to combine these constructions for a better result. The upper bound (provided both by Corollary 7 and the KőváriT. Sós-Turán Theorem) is $O\left(n^{4 / 3}\right)$.

We remark that, although not known if needed in Corollary 7 , the $n / \sqrt{m}$ term is meaningful. This is the threshold for a typical pair of symbols appearing together in many cyclic sequences. We need it for our estimate that not many more different than same pairs exist. If a typical pair of symbols appears together in only two cyclic sequences, it is possible that they only contribute different pairs. This happens in the above construction as well; since we construct linearly ordered (rather than cyclic) sequences that are pairwise intersection reverse, no "same pair" ever appears.

One can ask the same extremal question about linearly ordered sequences. Let $Q(n, m)$ stand for the maximum total length of $m$ pairwise intersection reverse sequences over an $n$ element alphabet. In this case two symbols cannot appear together in three sequences. The Kővári-T. Sós-Turán Theorem therefore gives the bounds $Q(n, m)=O\left(m n^{2 / 3}+n\right)$ and $Q(m, n)=O(n \sqrt{m}+m)$. For $m \leq n / \log ^{2} n$ or $m \geq n^{3}$ we get the same upper bounds that we $\operatorname{did}$ for $R(n, m)$. The upper bound for intermediate values of $m$ is shown by the dotted line in Figure 1. One gets simple constructions of intersection reverse sequences by 
considering set systems with pairwise intersection limited to singletons. Just as we noted in the case of cyclic sequences, this property ensures that the sequences are pairwise intersection reverse independent of the linear order chosen. The standard construction for such a set system is the set of lines in a finite plane, yielding $Q(n, m)=\Omega(n \sqrt{m})$ for $m \geq n$ and $Q(n, m)=\Omega(m \sqrt{n})$ for $m \leq n$. The bounds $Q(n, m) \geq n$ and $Q(n, m) \geq m$ are trivial. These bounds determine $Q(n, m)$ up to a constant factor for $m \leq n^{1 / 3}$ and $m \geq n$. Notice that the construction using parabolas in the plane yields pairwise intersection reverse linearly ordered sequences and so we have $Q(n, m)=\Omega\left(n^{5 / 6} m^{1 / 2}\right)$ for $n^{1 / 3} \leq$ $m \leq n^{2 / 3}$. Surprisingly, the "area of uncertainty" for $Q(n, m)$ is exactly the same parallelogram as it is for $R(n, m)$. Only when $n<m<n^{3}$ do the bounds for $Q(n, m)$ and $R(n, m)$ diverge. We do not know if allowing for cyclic sequences can yield longer intersection reverse collections in the $m<n$ case.

Problem 3. Does $R(n, m)=O(Q(n, m))$ hold for $m<n$ ?

As far as pseudo-circles are concerned, our result is conjectured to be far from optimal. The best known construction is a set of $n$ pseudo-circles that needs $\Omega\left(n^{4 / 3}\right)$ cuts before it becomes a collection of pseudo-segments. It is an important open problem to improve either bound on the minimum number of cuts that turn pseudo-circles into pseudo-segments.

\section{Acknowledgments}

We would like to thank János Pach for introducing us to this problem and for directing our attention to the many consequences that were shown in Section 3.

\section{References}

1. Pinchasi, R., Radoičić, R.: On the number of edges in geometric graphs with no self-intersecting cycle of length 4 . In Pach, J., ed.: Towards a Theory of Geometric Graphs. Number 342 in Contemp. Math., Providence, RI, Amer. Math. Soc. (2004)

2. Tamaki, H., Tokuyama, T.: How to cut pseudo-parabolas into pseudosegments. Discrete Comput. Geom. 19 (1998) 265-290

3. Aronov, B., Sharir, M.: Cutting circles into pseudo-segments and improved bounds for incidences. Discrete Comput. Geom. 28 (2002) 475-490

4. Agarwal, P., Nevo, E., Pach, J., Pinchasi, R., Sharir, M., Smorodinsky, S.: Lenses in arrangements of pseudo-circles and their applications. J. ACM 51 (2004) 139-186

5. Chan, T. M.: On levels in arrangements of curves. Discrete and Comput. Geom. 29 (2000) 375-393

6. Chan, T. M.: On levels in arrangements of curves, II: a simple inequality and its consequence. In: Proc. 44th IEEE Symposium on Foundations of Computer Science (FOCS). (2003) 544-550

7. Kővári, T., T. Sós, V., Turán, P.: On a problem of K. Zarankiewicz. Colloq. Math. 3 (1954) 50-57

8. Elekes, Gy.: Sums versus products in number theory, algebra and Erdös geometry. In G. Hálász, et. al., ed.: Paul Erdős and his mathematics II. Based on the conference, Budapest, Hungary, July 4-11, 1999. Volume 11 of Bolyai Soc. Math. Stud., Springer (2002) 241-290 